\title{
Platelet-rich plasma has beneficial effects in mice with osteonecrosis of the femoral head by promoting angiogenesis
}

\author{
SHICHAO TONG ${ }^{1}$, JIMIN YIN ${ }^{1}$ and JI LIU ${ }^{2}$ \\ ${ }^{1}$ Department of Orthopedics, Shanghai Sixth People's Hospital, Shanghai Jiaotong University, Shanghai 200231; \\ ${ }^{2}$ Department of Orthopedics, Ruijin Hospital of Shanghai Jiao Tong University School of Medicine, Shanghai 200025, P.R. China
}

Received April 13, 2016; Accepted March 17, 2017

DOI: $10.3892 /$ etm.2017.5655

\begin{abstract}
Platelet-rich plasma (PRP) is autologous and multifunctional. Platelet concentrate from blood contains highly concentrated platelets and various types of cells, including growth factors. PRP promotes the recovery of cell proliferation and differentiation. Osteonecrosis of the femoral head is a disease caused by femoral head damage or an insufficient blood supply, which leads to the death of bone cells and abnormal bone marrow composition. The subsequent repair of bone cells may result in changes to the structure of femoral head, femoral head collapse and joint dysfunction. PRP may promote the repair of injured articular cartilage in patients with joint diseases through the removal of harmful inflammatory factors. In the present study, the therapeutic effects and primary mechanism of PRP action were investigated using a glucocorticoid-induced femoral head osteonecrosis mouse model. Dexamethasone (DEX) and phosphate-buffered saline were used as controls. The therapeutic efficacy of PRP to treat osteonecrosis in murine femoral heads was evaluated by assessing clinical arthritis scores. The present study indicated that mice with osteonecrosis of the femoral head treated with PRP exhibited downregulated expression of interleukin (IL)-17A, IL-1 $\beta$, tumor necrosis factor- $\alpha$, receptor activator of nuclear factor $\kappa$-B ligand, IL- 6 and interferon- $\gamma$ in the inflammatory tissue. In addition, the levels of hepatocyte growth factor, intercellular adhesion molecule-1, osteopontin, platelet-derived endothelial cell growth factor, vascular endothelial growth factor, platelet-derived growth factor, insulin-like growth factor-1 and transforming growth factor- $\beta$ were increased following treatment with PRP. Joint tissue histological staining demonstrated that PRP alleviated osteonecrosis of the femoral head and reduced humoral and cellular immune responses that promoted beneficial effects on
\end{abstract}

Correspondence to: Professor Jimin Yin, Department of Orthopedics, Shanghai Sixth People's Hospital, Shanghai Jiaotong University, 600 Yishan Road, Shanghai 200231, P.R. China

E-mail: yinjiminpro@163.com

Key words: platelet-rich plasma, osteonecrosis of the femoral head, inflammatory factors, proangiogenic factors the histological parameters. Furthermore, the concentration of glucocorticoids were significantly decreased in the serum of PRP-treated mice with osteonecrosis compared with the DEX group $(\mathrm{P}<0.01)$. Notably, PRP promoted beneficial effects in mice with osteonecrosis of the femoral head by stimulating angiogenesis. Therefore, the present study indicated that treatment with PRP promotes beneficial effects by preventing joint inflammation, cartilage destruction and bone damage, and stimulating the repair of joint tissue in mice with osteonecrosis of the femoral head. These preclinical data suggest that PRP may be developed as a novel method of treating osteonecrosis of the femoral head.

\section{Introduction}

Osteonecrosis of the femoral head is an autoimmune disease manifesting primarily as a type of inflammatory arthritis that seriously threatens human health and quality of life $(1,2)$. The causes of femoral head necrosis are diverse and complex, which means that it is challenging to devise a classification system and determine the pathogenesis associated with this disease (3-5). Osteonecrosis of the femoral head generally occurs following trauma, or drug and alcohol abuse $(1,6)$. The symptoms of osteonecrosis of the femoral head include pain, stiff joints that limit activity, lameness and different degrees of femoral head necrosis $(2,7)$. In recent years, the incidence of osteonecrosis of the femoral head has increased and the majority of patients with osteonecrosis of the femoral head are now diagnosed in the clinic $(7,8)$. Currently, treatments for osteonecrosis of the femoral head include non-weight-bearing therapy, physical support, the promotion of osteoclast apoptosis and the reduction of osteoblast and osteocyte apoptosis (9-11). Therefore, further research into potential novel treatments of the disease is required.

Platelet-rich plasma (PRP) is a blood product that may be effective at treating osteonecrosis of the femoral head, cartilage disorders and rheumatoid arthritis (10). It has been demonstrated that PRP alleviates osteonecrosis of the femoral head without triggering side effects and treatment-related adverse events of PRP were not reported in a clinical investigation (12). The therapeutic outcomes of treatment with PRP, which is isolated from autologous peripheral blood mononuclear cells and is composed of cytokines, growth factors and other bio-active molecules, is efficient and innovative 
treatment protocols have been devised (13). Previous studies have demonstrated that intravenous injection of PRP repairs tendon and articular bone damage and contributes to the elimination of the inflammatory response; thus it may serve an important role on morphological properties and collagen microarchitecture (13-15). Furthermore, it has been suggested that PRP stimulates significant clinical improvements in patients with symptomatic osteoarthritis of the hip joints (16). Osteonecrosis of the femoral head is an intractable and chronic inflammatory autoimmune disease characterized by the release of inflammatory cytokines, which may promote degradative mediators, subsequent infiltration of autoimmune cells into the synovium and joint damage (17).

Previous studies have indicated that innate and adaptive immune responses have essential roles in regulating the expression of inflammatory cytokines, which target the synovium and subsequently result in the development of joint diseases $(17,18)$. Osteonecrosis of the femoral head is an autoimmune disease characterized by chronic inflammation of the joint (17). Furthermore, osteonecrosis of the femoral head is associated with chronic inflammation of the synovial joints, predominantly in the hands and feet, as well as systemic extra-articular inflammation (19). A previous study reported that the use of concentrated bone marrow aspirate and PRP resulted in significant pain relief and halted the progression of disease in the majority of patients with osteonecrosis of the femoral head (19). These data suggest that PRP may be effective at treating osteonecrosis of the femoral head.

In the present study, the efficacy and mechanism of PRP was investigated using a glucocorticoid-induced femoral head osteonecrosis mouse model. Inflammatory factors were analyzed in PRP, dexamethasone (DEX) and phosphate buffered saline (PBS)-treated groups. The therapeutic efficacy of PRP for mice with osteonecrosis of the femoral head was then evaluated by assessing clinical arthritis scores. The effects of PRP on the immune and inflammatory responses were also analyzed.

\section{Materials and methods}

Animal study. A total of 60 male C57BL/6J mice (6-8 weeks old; weighing 30-35 g) were purchased from Shanghai SLAC Laboratory Animals Co., Ltd., (Shanghai, China). All mice were housed in a temperature-controlled facility at $23 \pm 1^{\circ} \mathrm{C}$ with a relative humidity of $50 \pm 5 \%$ and a 12 -h light/dark cycle. All mice were identified by ear punching and were used to establish a glucocorticoid-induced $(100 \mathrm{mg} / \mathrm{kg})$ femoral head mouse model. The induction of glucocorticoid was achieved as described previously (20) via the subcutaneous injection of $100 \mathrm{mg} / \mathrm{kg}$ glucocorticoid (methylprednisolone, ModiQuest Research, Oss, The Netherlands) per mouse. The mice were divided into three groups and given a normal schedule and free access to a standard diet and water ( $n=20$ in each group). On day 7 after model establishment (visible dead in cartilage area and partial necrosis and collapse of the cartilage surface), mice received treatment with PRP (10 mg/kg; Sigma-Aldrich; Merck KGaA, Darmstadt, Germany), dexamethasone (DEX; $10 \mathrm{mg} / \mathrm{kg}$, Sigma-Aldrich; Merck KGaA) or the same volume of PBS by tail vein intravenous injection. Body weights of experimental mice were measured prior to each injection.
Treatments were conducted seven times, once every 3 days. All mice were decapitated under $50 \mathrm{mg} / \mathrm{kg}$ of intraperitoneal injection of sodium pentobarbital (3\%, Sigma-Aldrich; Merck $\mathrm{KGaA}$ ) on day 32 for histological analysis and measurement of spleen weight loss. Clinical femoral head osteonecrosis scores were evaluated using a scale of $0-2$, as previously described ( 0 , health; 0.5 , one leg disabled in action; 1.0 , two legs disabled in action; 1.5 , three legs disabled in action; and 2.0, four legs disabled in action) (21). The present study was performed in strict accordance with the recommendations of the Guide for the Care and Use of Laboratory Animals of the Shanghai Jiaotong University (Shanghai, China). All animal procedures were reviewed and approved by the Shanghai Jiaotong University Animal Care Committee. All surgeries and euthanasia were performed using sodium pentobarbital anesthesia and efforts were made to minimize the suffering of experimental mice.

ELISA. Interferon (IFN)- $\gamma$, interleukin (IL)-6, IL-17A, tumor necrosis factor (TNF)- $\alpha$, IL- $1 \beta$ and receptor activator of nuclear factor $\kappa-B$ ligand (RANKL) concentrations in the spleen of experimental mice were analyzed using ELISA. On day 32, spleens were harvested and homogenized in a commercialized buffer (Invitrogen; Thermo Fisher Scientific, Inc., Waltham, MA, USA). Subsequently, splenocytes were purified by centrifugation $(4 \times 1,000 \mathrm{x} \mathrm{g})$ at $4^{\circ} \mathrm{C}$ for $15 \mathrm{~min}$ and the homogenates were quantified using a cell counter (Vi-CELL XR; Beckman Coulter, Inc., Brea, CA, USA) according to previous study (20). IFN- $\gamma$ (ERIFNGALPHA), IL-6 (29-8061-65), IL-17A (EMIL17A), TNF- $\alpha$ (CMC3013), IL-1 $\beta$ (ERIL1B) and RANKL (EMTNFSF11) concentrations were measured using ELISA kits (Thermo Fisher Scientific, Inc.) according to the manufacturer's instructions. In addition, glucocorticoid serum concentration level was also analyzed using an ELISA kit (EIACORT; Bio-Rad Laboratories, Inc.) according to the manufacturer's instructions.

$R N A$ isolation and $R T-q P C R$. Total RNA from murine spleens and synovial cells was extracted using an RNA isolation kit (AM1912M; Invitrogen; Thermo Fisher Scientific, Inc.) according to the manufacturer's instructions. All gene primers were synthesized and listed in Table I. The concentration of RNA was quantified by spectrophotometry at $260 \mathrm{~nm}$ (Smart Spec TM 3000; Bio-Rad Laboratories, Inc.). Quantified RNA was subjected to reverse transcription into cDNA using a reverse transcription kit (Qiagen Sciences, Inc., Gaithersburg, MD, USA) and quality was confirmed by electrophoresis. cDNA was diluted 1:10 with distilled water and $10 \mu \mathrm{l}$ was used for amplification. Specific primer sets for IFN- $\gamma$, IL-6, IL-17A, VEGF, PDGF, IGF-1, TGF- $\beta$, TNF- $\alpha$, IL- $1 \beta$ and RANKL, HGF, ICAM-1, OPN and PD-ECGF were conserved in our lab. RT-qPCR was performed using a real-time quantitative PCR kit (Roche Molecular Diagnostics, Pleasanton, CA, USA) with a SYBR Green Master Mix system according to the protocol provided with the parameter-specific kits (K0241; Roche Diagnostics, Basel, Switzerland). A total of 45 amplification cycles were performed, including $94^{\circ} \mathrm{C}$ for $30 \mathrm{sec}$, denaturation at $96^{\circ} \mathrm{C}$ for $5 \mathrm{sec}$, primer annealing at $68^{\circ} \mathrm{C}$ for $5 \mathrm{sec}$ with touchdown to $58^{\circ} \mathrm{C}$ for $15 \mathrm{sec}$ and applicant extension at $72^{\circ} \mathrm{C}$ for $10 \mathrm{sec}$. Relative gene expression levels were calculated by 
Table I. Sequences of the primers used in the present study.

Sequence

\begin{tabular}{lll}
\cline { 2 - 3 } Gene name & \multicolumn{1}{c}{ Reverse } & \multicolumn{1}{c}{ Forward } \\
\hline IL-17A & 5'-ATGCACAGCCACCGCGACTT-3' & 5'-CTTCATGACTGCCTCCAAGTAG-3' \\
IL-1 $\beta$ & 5'-GGCTGCTTCCAAACCTTTGA-3' & 5'-GAAGACACGGATTCCATGGT-3' \\
TNF- $\alpha$ & 5'-TCCAGACTTCCTTGAGACA-3' & 5'-GGCGATTACAGACACAACT-3' \\
RANKL & 5'-AAGGCGAGAGATTCTTTCCCTG'-3 & 5'-ACTGGGGACAATTCACTAGAGC-3' \\
IL-6 & 5'-GTGAGGAACAAGCCAGAG-3' & 5'-TGACCAGAAGAAGGAATGC-3' \\
IFN- $\gamma$ & 5'-CACCCTCTGTCACCTGCTCAA-3' & 5'-ATGGCGCTGAGAAGACTTGGT-3' \\
$\beta$-actin & 5'-CGGAGTCAACGGATTTGGTC-3' & 5'-AGCCTTCTCCATGGTCGTGA-3'
\end{tabular}

IL, interleukin; TNF- $\alpha$, tumor necrosis factor- $\alpha$; IFN- $\gamma$, interferon- $\gamma$.

$2^{-\Delta \Delta \mathrm{Cq}}$ method (22). The results were presented as the $\mathrm{n}$-fold way compared with $\beta$-actin. All experiments were repeated three times.

Histopathological analysis. Mice with osteonecrosis of the femoral head were sacrificed using pentobarbital anesthesia $(50 \mathrm{mg} / \mathrm{kg}$ ) on day 32 and the hips joints were separated, and $4-\mu \mathrm{m}$ thick sections were fixed in $10 \%$ formalin for $12 \mathrm{~h}$ at $25^{\circ} \mathrm{C}$. The joints were subsequently decalcified and embedded in paraffin. The hips joints from experimental mice were stained with hematoxylin and eosin. The therapeutic effects of PRP, DEX and PBS were analyzed in mice with glucocorticoid-induced osteonecrosis of the femoral head. The severity of osteonecrosis of the femoral head in the joints was scored on a scale of $0-5$ ( 0 , no joint inflammation arbitrary unit; 1 , 1 joint inflammation arbitrary unit; 2, 2 joint inflammation in arbitrary unit; 3, 3 joint inflammation arbitrary unit; 4, 4 joint inflammation arbitrary unit; and 5, 5 joint inflammation arbitrary unit) as previously described (23).

Statistical analysis. All data were presented as mean \pm standard deviation of triplicate results. Unpaired data were analyzed using Student's t test and comparisons of data between multiple groups were conducted using analysis of one-way variance and a Tukey Kramer test. A Kaplan-Meier curve was used to estimate the survival rate during the 120-day long-term observation to analyze the survival rate. $\mathrm{P}<0.05$ was considered to indicate a statistically significant difference.

\section{Results}

Functional analysis of PRP in synovial cells and spleens of mice with glucocorticoid-induced osteonecrosis of the femoral head. To investigate the efficacy of PRP in mice with glucocorticoid-induced osteonecrosis of the femoral head, the concentration of inflammatory cytokines in murine synovial cells and spleens were measured. It was determined that the levels of mRNA and concentrations of IL-17A, TNF- $\alpha$, IL-1 $\beta$ and RANKL were significantly downregulated in the DEX group compared with the PBS control group in synovial cells $(\mathrm{P}<0.01$; Fig. 1A) and the spleen $(\mathrm{P}<0.01$; Fig. 1B) respectively. PRP treatment significantly reduced levels of mRNA and concentration of IL-17A, IL-1 $\beta$, TNF- $\alpha$ and RANKL in the PRP group compared with the DEX group in murine synovial cells and spleens, respectively ( $\mathrm{P}<0.01$; Fig. $1 \mathrm{~A}$ and $\mathrm{B})$, contributing to the remission of osteonecrosis of the femoral head. Furthermore, the mRNA expression levels and concentrations of IFN- $\gamma$ and IL- 6 were significantly upregulated in the DEX group compared with the PBS group in murine synovial cells and spleens, respectively ( $\mathrm{P}<0.05$; Fig. $1 \mathrm{C}$ and $\mathrm{D}$, respectively); moreover, PRP treatment significantly upregulated the mRNA expression levels and concentrations of IFN- $\gamma$ and IL- 6 in the PRP group compared with the DEX group in murine synovial cells and spleens, respectively $(\mathrm{P}<0.01$; Fig. $1 \mathrm{C}$ and $\mathrm{D}$, respectively). These results suggested that PRP treatment effectively suppressed inflammatory cytokines IL-17A, IL-1 $\beta$, TNF- $\alpha$ and RANKL and promoted advantageous inflammatory cytokines IFN $-\gamma$ and IL- 6 for mice with osteonecrosis of the femoral head.

Therapeutic effects of PRP in mice with glucocorticoid-induced osteonecrosis of the femoral head. In order to evaluate the efficacy of PRP on the state of illness development, the femoral head osteonecrosis mouse model was established. Experimental mice were injected with PRP, DEX or PBS. The arthritic score of mice in each group was recorded every 3 days. PRP treatment significantly ameliorated both the clinical symptoms and hind paw swelling, thus reducing arthritic scores of PRP-treated mice compared with those of the DEX-treated mice $(\mathrm{P}<0.01$; Fig. 2A). In addition, the therapeutic effects of PRP were evaluated according to the humoral glucocorticoid concentration. Serological levels of anti-glucocorticoid immunoglobulin ( $\mathrm{Ig}) \mathrm{G}$ prior to and following treatment were measured in experimental mice. The results demonstrated that a significant decrease in anti-glucocorticoid IgG serum levels occurred in PRP-treated mice compared with the DEX-treated group ( $\mathrm{P}<0.01$; Fig. $2 \mathrm{~B})$. Vascular endothelial growth factor (VEGF), platelet-derived growth factor (PDGF), insulin-like growth factor-1 (IGF-1) and transforming growth factor (TGF)- $\beta$ mRNA expression levels were significantly increased in the DEX group compared with the PBS group in the synovial cells $(\mathrm{P}<0.01$; Fig. 2C-F, respectively). Furthermore, PRP treatment significantly upregulated the mRNA expression levels of VEGF, 
A

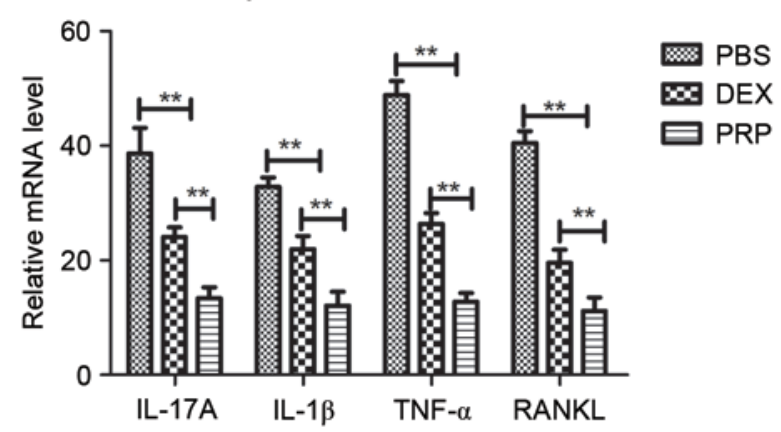

C

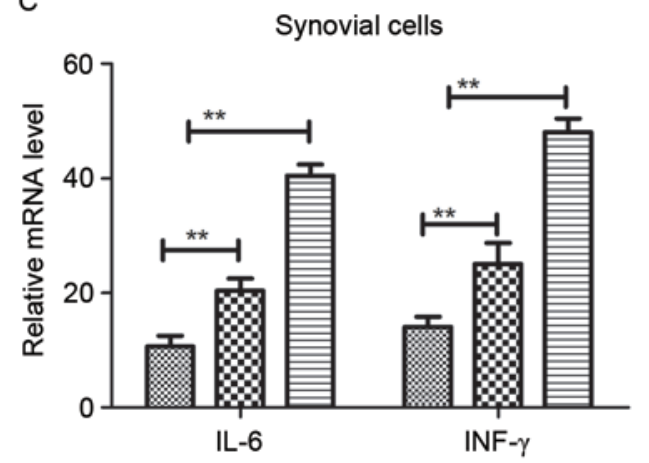

B

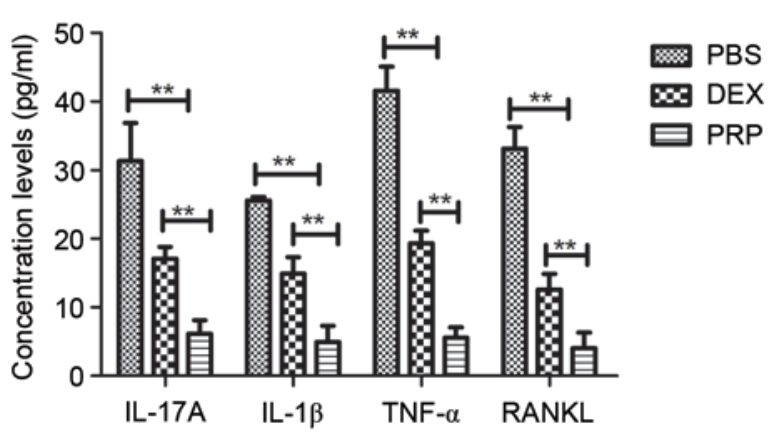

D

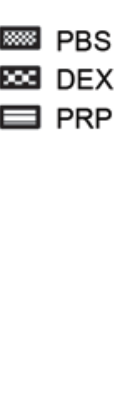

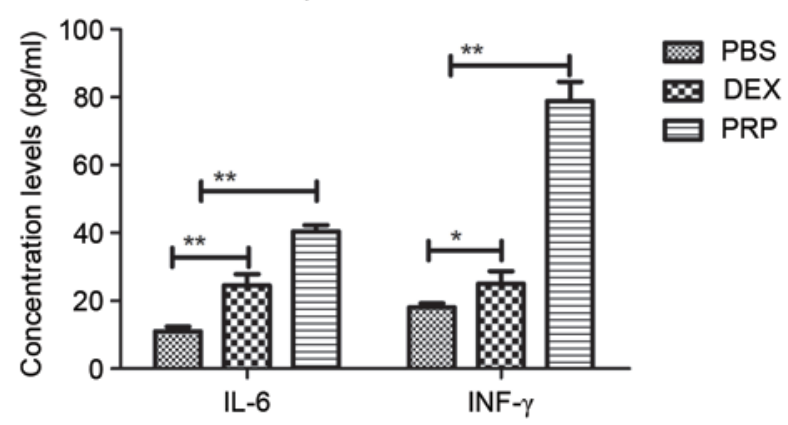

Figure 1. mRNA expression levels and concentration of inflammatory factors in spleens and synovial cells of mice with glucocorticoid-induced osteonecrosis of the femoral head. (A) IL-17A, TNF- $\alpha$, IL-1 $\beta$ and RANKL mRNA expression levels in synovial cells were analyzed by RT-qPCR. (B) IL-17A, TNF- $\alpha$, IL-1 $\beta$ and RANKL concentration levels in spleens were analyzed by ELISA. (C) IL-6 and IFN- $\gamma$ mRNA expression levels in synovial cells were analyzed by RT-qPCR. (D) IL-6 and IFN- $\gamma$ concentration levels in spleens were analyzed by ELISA. Data are expressed as mean \pm standard deviation. "P<0.05 and ${ }^{* *} \mathrm{P}<0.01$. RT-qPCR; reverse transcription-quantitative polymerase chain reaction; IL, interleukin; TNF- $\alpha$, tumor necrosis factor- $\alpha$; RANKL, receptor activator of nuclear factor $\kappa-\mathrm{B}$ ligand; IFN- $\gamma$, interferon- $\gamma$; PRP, platelet-derived plasma; DEX, dexamethasone; PBS, phosphate-buffered saline.

PDGF, IGF-1 and TGF- $\beta$ compared with the DEX group ( $\mathrm{P}<0.01$; Fig. 2C-F, respectively). These data suggest that PRP may induce the production of angiogenic factors, which may contribute to angiogenesis in femoral head osteonecrosis.

Body and spleen weight changes following PRP treatment. Body and spleen weight changes were evaluated following treatment with PRP DEX or PBS. The body weights of mice in the DEX group were significantly increased compared with the PBS group $(\mathrm{P}<0.01$; Fig. 3A) and PRP-treated mice exhibited a significantly increased body weight compared with the DEX group ( $\mathrm{P}<0.01$; Fig. 3A). It was determined that spleen weight loss in mice from the DEX group was significantly decreased compared with that of the PBS group $(\mathrm{P}<0.01)$ and that the spleen weight loss of mice in the PRP group was significantly decreased compared with that of the DEX group $(\mathrm{P}<0.01$; Fig. 3B). The DEX group exhibited significantly increased mRNA expression levels of hepatocyte growth factor (HGF; $\mathrm{P}<0.01$ ), intercellular adhesion molecule-1 (ICAM-1; $\mathrm{P}<0.01$ ), osteopontin (OPN; $\mathrm{P}<0.05)$ and platelet-derived endothelial cell growth factor ( $\mathrm{PD}-\mathrm{ECGF}$; $<0.05)$ compared with the PBS group (Fig. 3C-F, respectively). Furthermore, PRP treatment significantly increased the mRNA expression levels of HGF, ICAM-1, OPN and PD-ECGF compared with the DEX group $(\mathrm{P}<0.01$; Fig. $3 \mathrm{C}-\mathrm{F})$. The data suggest that PRP treatment significantly increased body weight and promoted the mRNA expression levels of angiogenesis-related factors.
PRP treatment is beneficial in mice with osteonecrosis of the femoral head. All synovial specimens from mice with femoral head osteonecrosis that were treated with PRP, DEX or PBS were investigated. Data indicated that hypertrophy and leukocyte infiltration was significantly reduced following 4 weeks treatment with PRP compared with the DEX group $(\mathrm{P}<0.01$; Fig. 4A and B). In addition, the therapeutic mechanism of PRP in synovial cells from mice with osteonecrosis of the femoral head was also analyzed. Histological staining indicated that PRP-treated mice with osteonecrosis of the femoral head exhibited markedly improved synovial hyperplasia, inflammatory cell influx, destruction of the cartilage and angiogenesis compared with the DEX group (Fig. 4C and D). Taken together, these preclinical data suggest that PRP may be an efficient agent for treatment of femoral head osteonecrosis.

\section{Discussion}

Osteonecrosis of the femoral head is a serious arthritic disease (24). It has been demonstrated that adjunct treatment methods for osteonecrosis of the femoral head, such as the combination of core decompression with bone morphogenic proteins, growth factors, bone grafting and stem cells produces effective results (25). PRP is a blood product that may be able to effectively treat osteonecrosis of the femoral head, cartilage disorders and rheumatoid arthritis (26). However, there have been few studies investigating the effectiveness of PRP for 


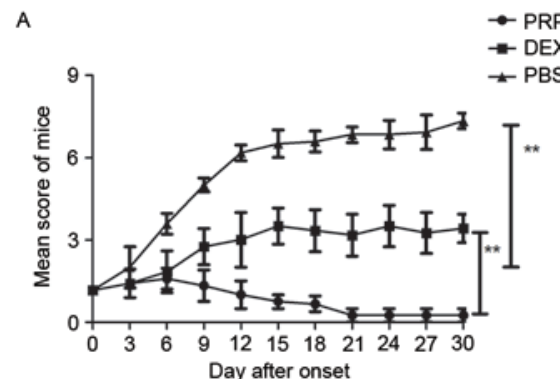

D

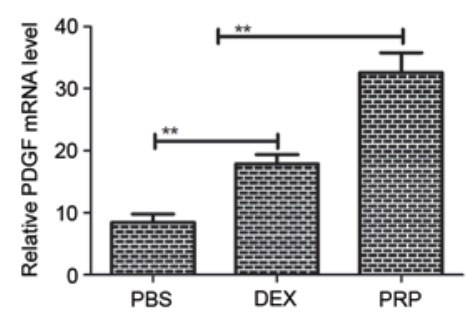

B

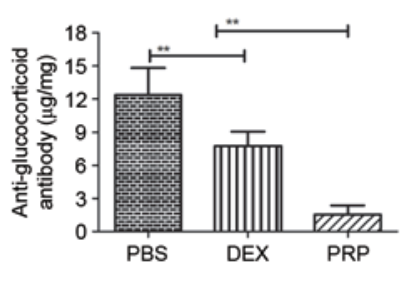

E

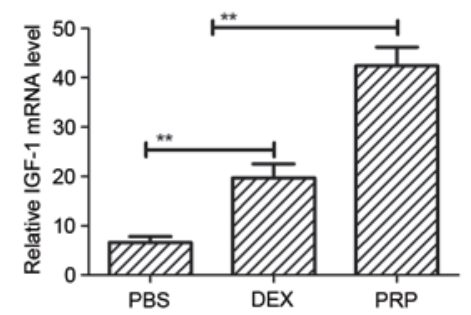

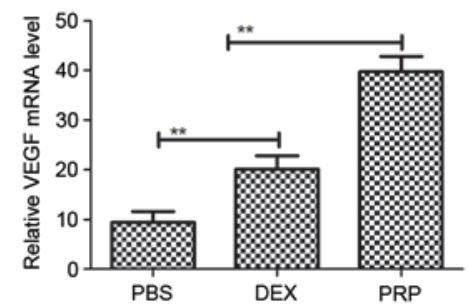

$\mathrm{F}$

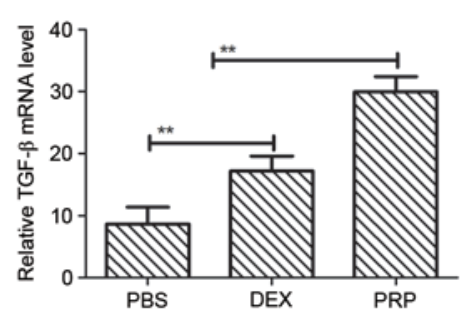

Figure 2. Therapeutic effects of PRP on mice with glucocorticoid-induced osteonecrosis of the femoral head. (A) Mice received subcutaneous injection of PRP $(10 \mathrm{mg} / \mathrm{kg})$, DEX $(10 \mathrm{mg} / \mathrm{kg})$ or the same volume of PBS. Arthritis scores were compared among non-treated $(\mathrm{n}=20)$, DEX $(\mathrm{n}=20)$ and PRP-treated $(n=20)$ groups during the course of treatment. Data are presented as the mean \pm standard deviation. (B) Serum concentrations of anti-glucocorticoid IgG in experimental mice were determined by ELISA. mRNA expression levels of (C) VEGF, (D) PDGF, (E) IGF-1 and (F) TGF- $\beta$ were detected in synovial tissue in experimental mice with osteonecrosis of the femoral head. Data are expressed as mean \pm standard deviation of triplicate samples. ${ }^{* *} \mathrm{P}<0.01$. PRP, platelet-derived plasma; DEX, dexamethasone; PBS, phosphate-buffered saline; VEGF, vascular endothelial growth factor; PDGF, platelet-derived growth factor; IGF-1, insulin-like growth factor-1; TGF- $\beta$, transforming growth factor- $\beta$.
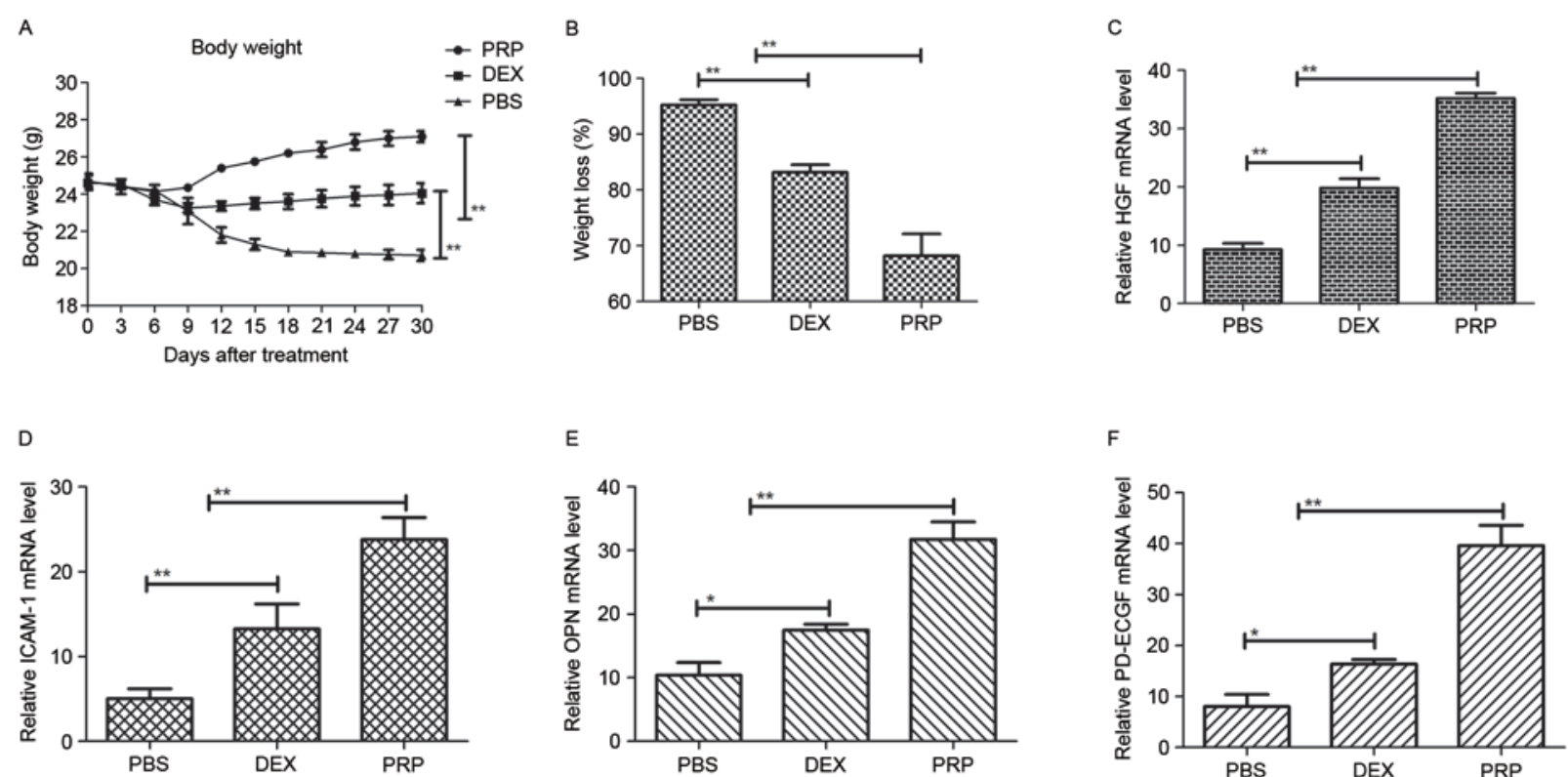

Figure 3. Effect of PRP treatment on body weight and proangiogenic factor expression. (A) Changes in body weight during treatment with PBS, DEX or PRP over a 30-day observation period. Data are expressed as mean \pm standard deviation. (B) Influence of PRP treatment on spleen weight loss in mice with osteonecrosis of the femoral head during the treatments. mRNA expression levels of (C) HGF, (D) ICAM-1, (E) OPN and (F) PD-ECGF were detected in murine spleens from mice with osteonecrosis of the femoral head. Results are expressed as the $n$-fold difference relative to $\beta$-actin. Data represent the mean \pm standard error of the mean of triplicate samples. "P<0.05 and ${ }^{* *} \mathrm{P}<0.01$. PRP, platelet-derived plasma; DEX, dexamethasone; PBS, phosphate-buffered saline; HGF, hepatocyte growth factor; ICAM-1, intercellular adhesion molecule-1; OPN, osteopontin; PD-ECGF, platelet-derived endothelial cell growth factor.

femoral head osteonecrosis therapy. The aim of the present study was to investigate the efficacy of PRP in the treatment of mice with glucocorticoid-induced osteonecrosis of the femoral head using a tail vein injection technique. The present study determined that repeated administration of PRP significantly relieved the pathogenesis of glucocorticoid-induced osteonecrosis of the femoral head. Moreover, despite the fact that IL- 6 and IFN- $\gamma$ have not been indicated as effective inhibitors of osteoclastic activity, the present results suggest that IFN- $\gamma$ and IL- 6 were upregulated following PRP treatment. Furthermore, mRNA expression levels of angiogenic factors including HGF, ICAM-1, OPN and PD-ECGF were 
A

Joint inflammation in experimental mice

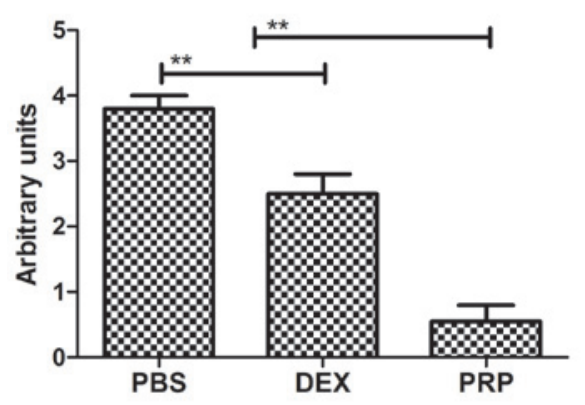

C

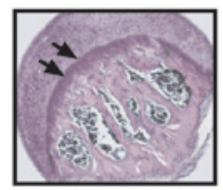

PBS

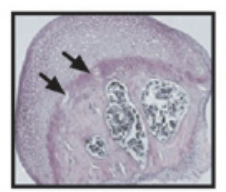

DEX

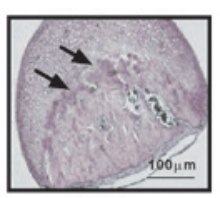

PRP

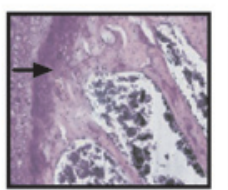

PBS

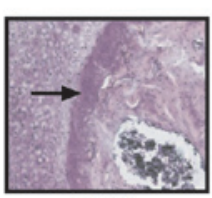

DEX

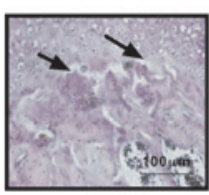

PRP

Figure 4. Histopathology of synovial grafts and femoral head in osteonecrosis of the femoral head mice. (A) PRP treatment reduced joint inflammation in mice with osteonecrosis of the femoral head. (B) PRP treatment reduced synovial degeneration in mice with osteonecrosis of the femoral head. (C) Analysis of destruction of the cartilage and angiogenesis revealed the effects of PRP in mice with osteonecrosis of the femoral head. Arrows indicated synovial hyperplasia and inflammatory cell influx. (D) Histological staining analysis indicated synovial hyperplasia and inflammatory cell influx in PRP-treated mice with osteonecrosis of the femoral head. Arrowheads indicated destruction of the cartilage. Data are expressed as mean \pm standard error of the mean ${ }^{* *} \mathrm{P}<0.01$. PRP, platelet-derived plasma; DEX, dexamethasone; PBS, phosphate-buffered saline.

significantly upregulated in PRP-treated mice. The present results also indicated that PRP treatment improved synovial hyperplasia, inflammatory cell influx, destruction of the cartilage and angiogenesis.

PRP was first discovered in 1993 and was identified to be rich in platelets, thus provoking its potential application in the field of biomedicine $(27,28)$. Current research has focused on the potential applications of PRP, as it contains high concentrations of PDGF, TGF- $\beta$, IGF, VEGF and epidermal growth factor $(29,30)$. Numerous studies have attempted to clarify the biological mechanism and effects of PRP on human diseases. However, the potency and limitations of PRP as a therapeutic agent to treat osteonecrosis of the femoral head has not been fully elucidated $(31,32)$. In the present study, the efficacy of PRP in a femoral head osteonecrosis mouse model was investigated and alterations in the expression of inflammatory and angiogenic factors were analyzed to determine the therapeutic outcomes.

Recently, PRP has exhibited favorable effects in various clinical joint disease cases (33). In addition, a previous report suggested that the efficacy and feasibility of PRP treatment for rheumatoid arthritis and avascular necrosis was marked (34). In the present study, the effectiveness of PRP at relieving pain and healing cartilage injuries were analyzed in PRP-treated mice $(n=20)$. The results of the present study indicate that PRP promotes significant pain relief and halts the progression of disease in the majority of mice with osteonecrosis of the femoral head.

Inflammatory cytokines have been identified as complex regulatory signal network involved in osteonecrosis of the femoral head and are mediated by different intracellular kinase signaling pathways to regulate the recruitment, stimulation, activation and performance of autoimmune cells (35). Although the causes of osteonecrosis of the femoral head are not fully understood, laboratory and clinical evidence suggests that inflammatory cytokines may contribute to its pathogenesis $(36,37)$. Theoretically, blocking inflammatory factor pathways may interrupt the inflammatory process to break the cycle of inflammation and limit joint damage $(38,39)$. The results of the present study suggest that the expression of inflammatory factors is decreased following PRP treatment, resulting in cartilage repair.

A previous study indicated that revascularization was beneficial in inducing the recovery of osteonecrosis of the femoral head and enhanced understanding regarding the mechanism of initiation, development and aggravation of osteonecrosis of the femoral head by revealing its role in mice with glucocorticoid-induced osteonecrosis of the femoral head (40). Hence, PRP may be beneficial in the treatment of osteonecrosis of the femoral head by promoting the expression of pro-angiogenic factors in spleen and synovial cells.

In the present study, PRP not only alleviated inflammation via humoral and cellular immune responses, but also led to beneficial effects, indicated by improvements in the histological parameters of joint tissue. These results were in accordance with those from a previous study concerning the pathology of osteonecrosis of the femoral head (41). In addition, since the overall PRP effect in the articular environment derives from interactions with the pre-existing environment 
and other cells, and as some surgical protocols involve the application of both platelets and cells, a number of studies have investigated the effect of PRP on mesenchymal stem cells of different origins $(42,43)$. Furthermore, the stimulatory effects of PRP treatment have been documented to promote proliferation and chondrogenic differentiation, which contributed to the production of IL-6 and IL-8 for the articular cartilage perform maintenance $(23,44)$.

Although previous studies have reported various direct effects of pharmacological agents on chondrocytes of the femoral head, it is essential to investigate the overall role of PRP on cytokine homeostasis of the entire joint. The data from the current study indicate that PRP significantly enhances synovial cell activity and improves synovial angiogenesis. In conclusion, the results of the current study indicate that PRP treatment for osteonecrosis of the femoral head in mice results in beneficial effects, as it alleviates joint inflammation, cartilage destruction and bone damage.

\section{Acknowledgements}

The present study was supported by the National Natural Science Foundation of China (grant no. 81501865).

\section{References}

1. Ma YW, Jiang DL, Zhang D, Wang XB and Yu XT: Radial extracorporeal shock wave therapy in a person with advanced osteonecrosis of the femoral head: A case report. Am J Phys Med Rehabil 95: e133-e139, 2016.

2. Lee GW, Park KS, Kim DY, Lee YM, Eshnazarov KE and Yoon TR: Results of total hip arthroplasty after core decompression with tantalum rod for osteonecrosis of the femoral head. Clin Orthop Surg 8: 38-44, 2016.

3. Meyers MH: Osteonecrosis of the femoral head. Pathogenesis and long-term results of treatment. Clin Orthop Relat Res: 51-61, 1988.

4. Gold EW and Cangemi PJ: Incidence and pathogenesis of alcohol-induced osteonecrosis of the femoral head. Clin Orthop Relat Res: 222-226, 1979.

5. Vignon $\mathrm{G}$ and Meunier P: Pathogenesis of primary osteonecrosis of the femoral head. Hypothesis of the subchondral fatigue fracture. Nouv Presse Med 2: 1751-1753, 1973 (In French).

6. Cabrolier J and Molina M: Is instillation of bone marrow stem cells at the time of core decompression useful for osteonecrosis of the femoral head? Medwave 16 (Suppl 1): e6406, 2016 (In Spanish).

7. Kianmehr N, Bidari A, Mofidi M and Bahar N: Silent osteonecrosis of the femoral head following high-dose corticosteroids in patients with systemic rheumatic diseases. Med J Islam Repub Iran 29: 259, 2015.

8. Jin H, Xu T, Chen Q, Wu C, Wang P, Mao Q, Zhang S, Shen J and Tong P: The fate and distribution of autologous bone marrow mesenchymal stem cells with intra-arterial infusion in osteonecrosis of the femoral head in dogs. Stem Cells Int 2016: 8616143, 2016.

9. Sadile F, Bernasconi A, Russo S and Maffulli N: Core decompression versus other joint preserving treatments for osteonecrosis of the femoral head: A meta-analysis. Br Med Bull 118: 33-49, 2016

10. Yamamoto $\mathrm{T}$ and Iwamoto $\mathrm{Y}$ : Corticosteroids treatments and osteonecrosis of the femoral head. Fukuoka Igaku Zasshi 106: 47-53, 2015.

11. Wang C, Peng J and Lu S: Summary of the various treatments for osteonecrosis of the femoral head by mechanism: A review. Exp Ther Med 8: 700-706, 2014.

12. Gibbs N, Diamond R, Sekyere EO and Thomas WD: Management of knee osteoarthritis by combined stromal vascular fraction cell therapy, platelet-rich plasma, and musculoskeletal exercises: A case series. J Pain Res 8: 799-806, 2015.

13. Vannini F, Di Matteo B and Filardo G: Platelet-rich plasma to treat ankle cartilage pathology-from translational potential to clinical evidence: A systematic review. J Exp Orthop 2: 2, 2015.
14. Wilson JJ, Lee KS, Chamberlain C, DeWall R, Baer GS, Greatens $M$ and Kamps N: Intratendinous injections of platelet-rich plasma: Feasibility and effect on tendon morphology and mechanics. J Exp Orthop 2: 5, 2015.

15. Zhang X, Wang J, Ren M, Li L, Wang Q and Hou X: A novel collagen/platelet-rich plasma (COL/PRP) scaffold: Preparation and growth factor release analysis. Cell Tissue Bank 17: 327-334, 2016.

16. Papavasiliou A, Yercan HS and Koukoulias N: The role of hip arthroscopy in the management of osteonecrosis. J Hip Preserv Surg 1: 56-61, 2014.

17. Hodge JA, Kawabata TT, Krishnaswami S, Clark JD, Telliez JB, Dowty ME, Menon S, Lamba M and Zwillich S: The mechanism of action of tofacitinib - an oral Janus kinase inhibitor for the treatment of rheumatoid arthritis. Clin Exp Rheumatol 34: 318-328, 2016.

18. van der Goes MC, Jacobs JW and Bijlsma JW: Rediscovering the therapeutic use of glucocorticoids in rheumatoid arthritis. Curr Opin Rheumatol 28: 289-296, 2016.

19. van der Jagt D, Mokete L, Pietrzak J, Zalavras CG and Lieberman JR: Osteonecrosis of the femoral head: Evaluation and treatment. J Am Acad Orthop Surg 23: 69-70, 2015.

20. Figueiredo CP, Simon D, Englbrecht M, Haschka J, Kleyer A, Bayat S, Hueber A, Pereira RM, Rech J and Schett G: Quantification and impact of secondary osteoarthritis in patients with anti-citrullinated protein antibodies positive rheumatoid arthritis. Arthritis Rheumatol 68: 2114-2121, 2016.

21. Zalavras CG and Lieberman JR: Osteonecrosis of the femoral head: Evaluation and treatment. J Am Acad Orthop Surg 22: 455-464, 2014.

22. Livak KJ and Schmittgen TD: Analysis of relative gene expression data using real-time quantitative PCR and the 2(-Delta Delta C(T)) method. Methods 25: 402-408, 2001

23. Bai F, Tian H, Niu Z, Liu M, Ren G, Yu Y, Sun T, Li S and Li D: Chimeric anti-IL-17 full-length monoclonal antibody is a novel potential candidate for the treatment of rheumatoid arthritis. Int J Mol Med 33: 711-721, 2014.

24. Pierce TP, Elmallah RK, Jauregui JJ, Poola S, Mont MA and Delanois RE: A current review of non-vascularized bone grafting in osteonecrosis of the femoral head. Curr Rev Musculoskelet Med 8: 240-245, 2015.

25. Pierce TP, Jauregui JJ, Elmallah RK, Lavernia CJ, Mont MA and Nace J: A current review of core decompression in the treatment of osteonecrosis of the femoral head. Curr Rev Musculoskelet Med 8: 228-232, 2015

26. Smyth NA, Haleem AM, Ross KA, Hannon CP, Murawski CD, Do HT and Kennedy JG: Platelet-rich plasma may improve osteochondral donor site healing in a rabbit model. Cartilage 7: 104-111, 2016.

27. Cenni E, Arciola CR, Ciapetti G, Granchi D, Savarino L, Stea S, Cavedagna D, Falsone G, Mari G and Pizzoferrato A: Assessment of the plasma phase of coagulation in platelet-rich plasma after passage through a special polyester filter. Minerva Medica 84: 589-593, 1993 (In Italian)

28. Leira R, Castillo J, Martinez F, Prieto JM and Noya M: Platelet-rich plasma serotonin levels in tension-type headache and depression. Cephalalgia 13: 346-348, 1993.

29. Donovan J: Successful treatment of corticosteroid-resistant ophiasis-type alopecia areata (AA) with platelet-rich plasma (PRP). JAAD Case Rep 1: 305-307, 2015.

30. Oz M, Cetinkaya N, Bas S, Korkmaz E, Ozgu E, Terzioglu GS, Buyukkagnici U, Akbay S, Caydere $\mathrm{M}$ and Gungor T: Response to the Letter to the Editor-Re: A randomized controlled experimental study of the efficacy of platelet-rich plasma and hyaluronic acid for the prevention of adhesion formation in a rat uterine horn model. Arch Gynecol Obstet 294: 667, 2016.

31. Yilmaz S, Aksoy E, Doganci S, Yalcinkaya A, Diken AI and Cagli K: Autologous platelet-rich plasma in treatment of chronic venous leg ulcers: A prospective case series. Vascular 23: 580-585, 2015.

32. Malahias MA, Chytas D, Babis GC and Nikolaou VS: Platelet-rich plasma guided injections: Clinical application in peripheral neuropathies. Front Surg 1: 41, 2014.

33. Reghini MF, Ramires Neto C, Segabinazzi LG, Castro Chaves MM, Dell'Aqua Cde P, Bussiere MC, Dell'Aqua JA Jr, Papa FO and Alvarenga MA: Inflammatory response in chronic degenerative endometritis mares treated with platelet-rich plasma. Theriogenology 86: 516-522, 2016. 
34. San Sebastian KM, Lobato I, Hernández I, Burgos-Alonso N, Gomez-Fernandez MC, López JL, Rodríguez B, March AG, Grandes $\mathrm{G}$ and Andia I: Efficacy and safety of autologous platelet rich plasma for the treatment of vascular ulcers in primary care: Phase III study. BMC Fam Pract 15: 211, 2014.

35. Lebouvier A, Poignard A, Cavet M, Amiaud J, Leotot J, Hernigou P, Rahmouni A, Bierling P, Layrolle P, Rouard H and Chevallier N: Development of a simple procedure for the treatment of femoral head osteonecrosis with intra-osseous injection of bone marrow mesenchymal stromal cells: Study of their biodistribution in the early time points after injection. Stem Cell Res Ther 6: 68, 2015.

36. Chiu WC, Lai YP and Chou MY: Humanization and characterization of an anti-human TNF- $\alpha$ murine monoclonal antibody. PloS One 6: e16373,2011.

37. Weisman MH: TNF and anti-TNF treatment in rheumatoid arthritis (RA). What we know and what we still need to know. Ryumachi 37: 142-143, 1997.

38. Elliot MJ, Maini RN, Feldmann M, Long-Fox A, Charles P, Katasikis P, Brennan FM, Bijl H, Ghrayeb J and Woody JN: Treatment of rheumatoid arthritis with chimeric monoclonal antibodies to tumor necrosis factor alpha. Arthritis Rheum 58 (2 Suppl): S92-S101, 2008

39. Segal B, Rhodus NL and Patel K: Tumor necrosis factor (TNF) inhibitor therapy for rheumatoid arthritis. Oral Surg Oral Med Oral Pathol Oral Radiol Endod 106: 778-787, 2008.
40. Wang B, Geng Q, Hu J, Shao J, Ruan J and Zheng J: Platelet-rich plasma reduces skin flap inflammatory cells infiltration and improves survival rates through induction of angiogenesis: An experiment in rabbits. J Plast Surg Hand Surg 50: 239-245, 2016.

41. Kalyam K, Kavoussi SC, Ehrlich M, Teng CC, Chadha N, Khodadadeh S and Liu J: Irreversible blindness following periocular autologous platelet-rich plasma skin rejuvenation treatment. Ophthal Plast Reconstr Surg 33 (3S Suppl 1): S12-S16, 2016.

42. Choy EH and Panayi GS: Cytokine pathways and joint inflammation in rheumatoid arthritis. N Engl J Med 344: 907-916, 2001.

43. Hermeto LC, DeRossi R, Oliveira RJ, Pesarini JR, Antoniolli-Silva AC, Jardim PH, Santana AE, Deffune E, Rinaldi JC and Justulin LA: Effects of intra-articular injection of mesenchymal stem cells associated with platelet-rich plasma in a rabbit model of osteoarthritis. Genet Mol Res 15, 2016.

44. Suzuki M, Tetsuka T, Yoshida S, Watanabe N, Kobayashi M, Matsui $\mathrm{N}$ and Okamoto T: The role of p38 mitogen-activated protein kinase in IL- 6 and IL-8 production from the TNF-alphaor IL-1beta-stimulated rheumatoid synovial fibroblasts. FEBS Lett 465: 23-27, 2000

(i) This work is licensed under a Creative Commons Attribution-NonCommercial-NoDerivatives 4.0 International (CC BY-NC-ND 4.0) License. 